\title{
Solvent Content in Thin Spin-Coated Polystyrene Homopolymer Films
}

\author{
J. Perlich, ${ }^{\dagger}$ V. Körstgens,${ }^{\dagger}$ E. Metwalli, ${ }^{\dagger}$ L. Schulz, ${ }^{\dagger \dagger}$ R. Georgii, ${ }^{\S}$ and \\ P. Müller-Buschbaum $*,+$
}

Physik-Department E13, Technische Universität München, James-Franck-Str. 1, 85747 Garching, Germany, Département de Physique, Université Fribourg, Chemin Du Musée 3, 1700 Fribourg, Switzerland, and Forschungsneutronenquelle Heinz, Maier-Leibnitz, Technische Universität München, Lichtenbergstr. 1, 85747 Garching, Germany

\begin{abstract}
The solvent content of thin polystyrene (PS) films, spin-coated from protonated and deuterated toluene onto silicon substrates, is investigated. Neutron reflectometry (NR) is used to probe the total remaining solvent inside the PS films in a nondestructive and noninvasive way. In freshly prepared films, the investigated parameters are the molecular weight of PS and the total film thickness. Moreover, the effect of postproduction treatment by annealing at temperatures below and above the glass transition of PS as well as long-term storage over 2 years are examined to deduce the reduction of the remaining solvent. The remaining solvent content increases with increasing molecular weight and with increasing film thickness. An enrichment of toluene at the $\mathrm{Si} /$ polymer interface is found. Under the different annealing and storage conditions tested, the remaining solvent is not totally removed. The observed behavior is discussed in the framework of polymer thin films and compared with results obtained by alternative experimental approaches.
\end{abstract}

\section{Introduction}

Spin-coating is a widely used technique for the preparation of thin polymer films on top of solid substrates. ${ }^{1-4}$ Films with a very uniform thickness and small surface roughness can be prepared depending on the polymer/solvent combination used. Controlled by the preparation parameters, film thicknesses in a wide range from the submonolayer regime up to several microns can be prepared with spin-coating. 5

The spin-coating procedure starts with the deposition of an excess amount of solution on the substrate. Immediately after deposition, the solvent starts to evaporate and the concentration of the polymer in the solution naturally increases. Thereafter, the substrate is accelerated to the desired rotation speed, whereas the acting adhesive forces at the liquid/substrate interface and the centrifugal forces result in strong shearing of the liquid. Hence, the majority of the polymer solution is rapidly ejected from the substrate by a radial liquid flow. In the final stage of spinning at constant rate, the thinning of the film is dominated by solvent evaporation. The evaporation process causes the polymer concentration to increase more rapidly at the liquid/vapor interface, and thus a concentration gradient is formed through the liquid film. After the evaporation of most of the solvent, a uniform, practically solid polymer film forms. ${ }^{6-9}$ However, the freshly spin-coated polymer film consists of the polymer in a nonequilibrated state and a residual amount of solvent. In other words, because of the very short time scale on which the film forms, the morphology of the polymer chains is frozen and solvent is retained. ${ }^{10-12}$ Thus, the rapid absence of the majority of the solvent prevents the motion of the chains. However, it is well known that the actual remaining solvent content in thin polymer films has an effect on the chain mobility and film homogeneity. ${ }^{13-17}$ Consequently, the drying process is determined by the film's ability to retain the solvent and the diffusion of solvent molecules and the polymer chains. Practically, the

\footnotetext{
* Corresponding author. Tel: +49 89289 12451; Fax: +49 8928912473 ; E-mail: muellerb@ph.tum.de.

† Physik-Department E13, Technische Universität München.

Université Fribourg.

${ }^{\S}$ Forschungsneutronenquelle Heinz Maier-Leibnitz, Technische Universität München.
}

solvent evaporation influences the thin film roughness, which occurs for the solid film. ${ }^{18-20}$ In terms of long-term stability, the reduction of the solvent content gives an estimate of possible aging effects. ${ }^{21}$ Furthermore, the solvent content influences the charge carrier transport, adhesion strength of adhesives, and most importantly, the glass-transition temperature, $T_{\mathrm{g}}{ }^{22-27}$ Because the latter is found to be dependent on the film thickness and substrate interaction, it might be expected that the solvent content depends on the film thickness as well. ${ }^{28}$ This explanation shows that many effects cannot be decoupled but rather exhibit a complex interplay among each other.

Several groups have investigated the solvent content in thin spin-coated homopolymer as well as block copolymer films with the focus on application-related questions. Only a few authors address the actual remaining solvent content in thin spin-coated films from a fundamental perception. ${ }^{29-34}$ Among them, GarcíaTuriel and Jérome studied the actual solvent retention in thin polymer films by gas chromatography (GC). ${ }^{35}$ They selected polystyrene (PS) with a molecular weight of $M_{\mathrm{w}}=178 \mathrm{~kg} / \mathrm{mol}$ and toluene as a model system and prepared thin homopolymer films in a thickness range of $15-500 \mathrm{~nm}$. Before analyzing the films with GC, all samples were annealed at a temperature of $115^{\circ} \mathrm{C}$ for $6 \mathrm{~h}$. It was found that the relative amount of retained toluene decreases from 35 to $\sim 2 \%$ with increasing film thickness. For film thicknesses of $100 \mathrm{~nm}$ and above, the relative amounts of retained solvent in the film are on the order of a few percent or less. In addition, from these results, they conclude that the solvent is retained at the polymer/substrate interface. Such an enrichment effect at the substrate interface was also reported by others. ${ }^{36-38}$ Nevertheless, the study of García-Turiel and Jérome gives quantitative information on the absolute amount of solvent in thin polymer films, which is hardly found elsewhere. However, for GC analysis, the spin-coated films were redissolved and the obtained solutions were analyzed with respect to the solvent used for the spin-coated films. In other words, the analysis with GC is destructive. Hence, the aim of the presented investigation is to apply a nondestructive technique to determine the amount of solvent remaining, which enables a successive characterization as well as further application of the spin-coated films. In this regard, neutrons meet the desired needs of a nondestructive, noncontact, and sensitive probe. ${ }^{39-42}$ 
Previously, our group extensively studied swelling and deswelling kinetics of thin PS films with in situ neutron reflectometry (NR). ${ }^{43,44}$ The focus of these investigations was on tracing changes in the film thickness due to swelling upon exposure to a saturated as well as undersaturated toluene atmosphere. The thickness change upon exposure was due to an uptake of toluene. The starting point of such swelling experiments was the initially prepared polymer film. Such an initial dry deuterated PS (dPS) film with, for example, a thickness of $49.8 \mathrm{~nm}$ (prior to exposure) already showed a solvent content of $\sim 7 \mathrm{v} / \mathrm{v} \%$.

The approach presented within this investigation solely focuses on the remaining solvent content in thin spin-coated homopolymer films. To the best of our knowledge, this is the first neutron reflectivity investigation of freshly prepared homopolymer films without any further processing after the spincoating with the primary aim of obtaining quantitative information on the remaining solvent content. In this regard, Kanaya et al. also investigated PS thin films with NR, but with the focus on annealing effects at different film thicknesses, whereas the role of the remaining solvent was not discussed. ${ }^{45}$

Our model system consists of thin spin-coated PS films on silicon substrates. For this investigation various molecular weights of PS are selected, and by simply varying the concentration of the polymer in solution, different film thicknesses are realized. The selection of both parameters for a polymer results in a well-defined thin polymer film. Needless to say, the spin-coating conditions, for example, acceleration and rotation speed, significantly influence the film thickness. Under these conditions and because of the fact that both parameters are easily accessible in the sense of traceability, the solvent content is studied depending on film thickness and molecular weight. ${ }^{46}$ In this regard, as already stated, the film thickness significantly influences $T_{\mathrm{g}}$, and the same trend is expected for the solvent content. Moreover, the preparation of thin films of constant thickness from polymers of different molecular weights is expected to influence the free volume in the thin film. ${ }^{47-51}$ As a consequence, more unoccupied volume is available where more solvent might be retained.

After spin-coating, the fresh samples are stored in a box while no measurements are performed. For the investigation of fresh samples, it is essential to minimize the time between the final preparation and the NR measurement itself. With appropriate time management, in this investigation, we managed to minimize the storage time before the NR measurement to $\sim 30$ min, taking into account the time necessary to align the sample properly. After the successful NR measurement, the samples are further characterized, for example, by the use of X-ray reflectometry (XRR), to provide all necessary information for the final extraction of the solvent content from the NR data.

After a brief introduction to the sample preparation and the fundamentals of the experimental techniques (XRR and NR), the obtained solvent content dependence on PS molecular weight and PS film thickness are discussed. Next, annealing and storage conditions are investigated, and the solvent profile along the surface normal is explored. The article concludes with a summary of the recent results.

\section{Experimental Section}

2.1. Sample Preparation. The homopolymer used in this investigation was protonated PS that was polymerized anionically (Polymer Standard Source, Mainz, Germany). Different molecular weights of PS of $M_{\mathrm{w}}=7,27.5,207,514,712,908$, and $1530 \mathrm{~kg} /$ mol and a typical narrow molecular weight distribution of $M_{\mathrm{w}} / M_{\mathrm{N}}$ $=1.03$ were selected. The solvents taken for the preparation of the homopolymer solution were protonated toluene $\left(\mathrm{C}_{7} \mathrm{H}_{8}\right.$, Carl
Roth, purity $\geq 99.9 \%)$ and deuterated toluene $\left(\mathrm{C}_{7} \mathrm{D}_{8}\right.$, deutero, purity $99.5 \%)$, denoted toluene and toluene- $d_{8}$, respectively.

The polymer films were prepared on top of precleaned Si (100) wafers with a very thin native oxide layer. Cleaning of the substrates was performed in an acidic bath $\left(160 \mathrm{~mL} \mathrm{H}_{2} \mathrm{SO}_{4}, 70 \mathrm{~mL} \mathrm{H}_{2} \mathrm{O}_{2}\right.$, and $110 \mathrm{~mL}$ deionized water) at a temperature of $80{ }^{\circ} \mathrm{C}$ for 15 min. ${ }^{52}$ Subsequently, we removed the remaining acid from the substrates by thoroughly rinsing them with deionized water; finally, the wafers were dried with compressed nitrogen.

The sample solutions were prepared according to the following procedure: The amount of PS homopolymer was calculated by an empirical formula that can be found elsewhere ${ }^{53}$ to obtain the desired film thickness and hence provide the required concentration. The concentration was dependent on the PS molecular weight as well as the spin-coating parameters. After the addition of the solvent to the PS, the solution was stirred for a sufficient amount of time to dissolve the polymer completely.

The PS films were prepared by spin-coating the individual solutions for $30 \mathrm{~s}$ at $2000 \mathrm{rpm}$ rotation speed. A circular shape of the Si wafers was used instead of a rectangular shape. The circular shape is optimal for the substrate to obtain homogeneously spreadout films because the distance to the edges of the wafers is equidistant to the center of rotation. Therewith, a steady flow of the solution during the spin-coating was supplied in all outward directions. In contrast, for rectangular-shaped samples, the flow is restricted by the nonequidistant distance to the edges, resulting in the optically visible spin-coating ellipse. This ellipse corresponds to variations in the film thickness, whereas a homogeneous area is found only in the center of the substrate. On the circular-shaped substrates used, a uniform film thickness was found over the entire wafer.

Three different sample series were prepared: The first series was composed of thin films of fixed thickness of $d=50 \mathrm{~nm}$ and different PS molecular weight $\left(M_{\mathrm{w}}=7,27.5,207,514,712,908\right.$, and 1530 $\mathrm{kg} / \mathrm{mol})$. The second series was films of different thicknesses $(d=$ $10-500 \mathrm{~nm})$ of fixed PS molecular weight $\left(M_{\mathrm{w}}=207 \mathrm{~kg} / \mathrm{mol}\right)$. For both series the samples were immediately tested after preparation to obtain information about freshly prepared films. In a third series, the aging behavior was addressed. At a fixed PS molecular weight $\left(M_{\mathrm{w}}=207 \mathrm{~kg} / \mathrm{mol}\right)$ and fixed film thickness, samples were annealed for $8 \mathrm{~h}$ under vacuum below the PS glass-transition temperature, $T_{\mathrm{g}}$, at $70{ }^{\circ} \mathrm{C}$ and above the $T_{\mathrm{g}}$ at 120 and $160{ }^{\circ} \mathrm{C}$. Moreover, in a long-term experiment, one untreated sample was simply stored under ambient conditions and was revisited over 2 years.

For all series, one set of samples was prepared from deuterated toluene and one from protonated toluene, giving rise to two sets per series. The preparation of identical samples from protonated as well as deuterated solvent enables a direct comparison and the determination of the remaining solvent.

Please note that instead of the acceleration and rotation speed the concentration of PS was adjusted to obtain the desired film thickness and to operate the spin-coater with fixed parameters. The effective film thickness, $d_{\text {eff }}$, was determined by XRR after the timesensitive NR investigation and was cross-checked with white light interferometry.

2.2. Neutron Reflectometry. NR measurements were performed on the instrument MIRA at the neutron source FRM-II in Garching (Germany). ${ }^{54}$ MIRA is established for research with very cold neutrons (VCN), thereby giving access to large wavelengths in a range of $8 \leq$ $\lambda \leq 30 \AA$. The differential neutron flux of the sample is on the order of $5 \times 10^{5}$ neutrons $/\left(\AA \cdot \mathrm{s} \cdot \mathrm{cm}^{2}\right)$ at a wavelength of $\lambda=10 \AA$. The use of large neutron wavelengths is advantageous for the detection of the position of the critical edge in NR because with increasing wavelength, the value of the critical angle $\alpha_{c}$ increases as

$$
\alpha_{c}=\lambda \sqrt{\frac{N b}{\pi}}
$$

where $\mathrm{Nb}$ is the coherent scattering length density (SLD) step at the interface. Correspondingly, the position of the critical edge shifts 
toward larger values of the scattering vector component along the surface normal

$$
q_{z}=\frac{2 \pi}{\lambda}\left(\sin \alpha_{\mathrm{i}}+\sin \alpha_{\mathrm{f}}\right)
$$

with the incident and exit angle $\alpha_{\mathrm{i}, \mathrm{f}}$. Thus, the resolution is increased for large neutron wavelengths. For the measurements, the desired wavelength was selected using a monochromator. Depending on the wavelength, either multilayer mirrors $(\lambda>8 \AA)$ or a mica monochromator $(7.7 \leq \lambda \leq 14 \AA$ ) was employed. The selected wavelength was fixed in the NR measurements with a resolution of $\Delta \lambda / \lambda=0.03$. Typically, a narrow range in the region of total external reflection was probed $\left(0.003 \leq q_{z} \leq 0.02 \AA^{-1}\right)$.

The NR setup used was composed of a horizontal scattering geometry with a vertically positioned sample. The sample and detector were moved in a $\theta / 2 \theta$ mode with an angular resolution of $\Delta \theta=0.01^{\circ}$ (reflectivity scan with $\alpha_{i}=\alpha_{\mathrm{f}}$ ) and a conventional ${ }^{3} \mathrm{He}$-counter was used as a detector.

The above-described sample preparation procedure enabled us to obtain sufficiently large samples to provide a large surface area for NR, hence maximizing the count rate at the detector and improving statistics. Most importantly, overillumination of the sample due to the rather large beam footprint at small incident angles was avoided because of the large sample size. As a result, the total reflection maximum exhibited a nice plateau of constant intensity (i.e., intensity independent of incident angle) cutoff by the distinct critical edge under determination.

For the data treatment, the raw measurement data represented by $I_{\mathrm{d}}(2 \theta)$ at the detector were first normalized by the corresponding incoming neutron flux $I_{\mathrm{m}}(t)$ during the counting time needed to measure $I_{\mathrm{d}}(2 \theta)$. Finally, $I_{\mathrm{d}}(2 \theta) / I_{\mathrm{m}}(t)$ was transformed to $R\left(q_{z}\right)$, which is the neutron reflectivity normalized to total reflection. The reflectivity data $R\left(q_{z}\right)$ were analyzed using the reflectivity simulation and analysis software programs Parratt and Motofit. ${ }^{55,56}$

In several beamtimes, the NR measurements of samples prepared under identical conditions were repeated to gain a statistical error and rule out experimental mistakes.

2.3. X-ray Reflectometry. XRR measurements were performed with a reflectometry-upgraded Siemens D5000 powder diffractometer. The upgrade included a special sample stage with a vacuum chuck, a tantalum knife edge collimator, and an automatic beam absorber, enabling the instrument to perform high-quality reflectivity measurements. The X-ray beam had a wavelength $\lambda=1.54 \AA(\mathrm{Cu}$ $\mathrm{K}_{\alpha}$ line). The beam was collimated with a slit system to run the experiments with a suitable beam divergence. The beam width was set to $12 \mathrm{~mm}$. A monochromator (graphite) was installed in the reflected beam path in front of the detector (scintillation counter) to filter the $\mathrm{Cu}-\mathrm{K}_{\beta}$ line as well as the fluorescence originating from the sample. An installed automatic absorber reduced the intensity by a factor of about 100, preventing the saturation of the detector and hence large errors in the counting rates. For XRR, this enabled the measurement in the range of total reflection, whereas beyond this range, the measurement was automatically continued without the absorber, avoiding manual operation and interruption of the experiment. The goniometer stage was operated at a resolution of $\Delta \theta=0.01^{\circ}$, corresponding to a resolution in reciprocal space of $\Delta q_{z}=0.0007 \AA^{-1}$.

To avoid bending stress of the thin $\mathrm{Si}$ wafer induced by the vacuum chuck, the samples were first fixed to a thick glass plate, which was then mounted on the vacuum chuck. This procedure is crucial for a precise XRR measurement because under the geometric setup conditions for the X-ray beam, a bent thin film sample appears to have a film of nonuniform thickness. As a result, in the reflectivity curve, all uniform-thickness-related features disappear, hence making the thickness determination impossible.

The XRR measurements were performed in the range of $0 \leq q_{z}$ $\leq 0.426 \AA^{-1}\left(0 \leq \theta \leq 3^{\circ}\right)$ in reciprocal space for each sample that had been investigated with NR beforehand. The measured raw data $I(2 \theta)$ were transformed to $R\left(q_{z}\right)$ (normalized reflectivity), and the data treatment to extract the thickness of the thin film and the roughness of stack interfaces was also performed with the reflectivity simulation and analysis software programs Parratt and Motofit. $^{55,56}$

2.4. Optical Microscopy. The sample surfaces were observed via optical microscopy using a Zeiss Axiotech $25 \mathrm{H}$ optical microscope with a magnification between 5 and 100. A Hitachi KP-D50 CCD camera recorded the micrographs.

\section{Results and Discussion}

The presented investigation of the remaining solvent content in thin spin-coated polymer films relies on the fact that the investigated component of the model system, namely, the solvent toluene, can be substituted by the deuterated solvent toluene- $d_{8}$ without any change in the physical properties of the thin spincoated PS films. Hence, regardless of the protonation or deuteration, both thin films will behave identically. However, the deuteration of the investigated solvent component results in a distinct differentiation in the SLD from all other involved materials, such as PS and the Si substrate. A measure of the contrast between different materials is represented by the ratio of the SLDs for the protonated solvent and the protonated PS film $\mathrm{SLD}_{\text {tol }} / \mathrm{SLD}_{\mathrm{PS}}=0.946 / 1.416=0.67$ and for the deuterated solvent and the protonated PS film $\mathrm{SLD}_{\text {tol- } d_{8}} / \mathrm{SLD}_{\mathrm{PS}}=5.6 / 1.416$ $=3.95$. Therefore, the highest contrast of PS and deuterated toluene in SLD is utilized to make the amount of residual solvent inside the thin film detectable.

The analysis of the NR data in the narrow range around the critical edge is based on a single-layer model, represented by the thin PS film, on a silicon substrate. ${ }^{45}$ The very thin native oxide layer present after cleaning has not been taken into account because NR data from freshly cleaned blank silicon substrates can be simulated solely by bare silicon.

Therefore, to match the simulated curve with the measured curve of the thin PS film, only the SLD of the PS is adjusted, whereas the magnitude of the adjustment represents a measure of the ratio of PS homopolymer to toluene- $d_{8}$ solvent. Because the relation $\mathrm{SLD}_{\text {tol }}<\mathrm{SLD}_{\mathrm{PS}}<\mathrm{SLD}_{\text {tol-d8 }}$ applies to the model system and because the film is a mixture of PS and toluene- $d_{8}$, the SLD for pure toluene- $d_{8}$ would mark the upper limit for this adjustment. In other words, reaching this limit would correspond to a film consisting solely of deuterated solvent.

The obtained SLD for the best model fit is used to determine the volume fraction $\Phi$ of remaining solvent with

$$
\Phi=\frac{\mathrm{SLD}-\mathrm{SLD}_{\mathrm{PS}}}{\mathrm{SLD}_{\mathrm{tol}-d_{8}}-\mathrm{SLD}_{\mathrm{PS}}}
$$

Consequentially, an increase in the probed SLD and hence a shift in the critical edge toward larger $q_{z}$ values corresponds to an increase in the amount of retained solvent inside the thin spin-coated PS films.

Therefore, the measured NR represents the average over the entire film, and thus the narrow $q_{z}$ scans provide the effective total solvent content $\phi$ (calculated from the volume fraction $\Phi$ ) of the thin film. In the following subsections, the results of the solvent content of thin spin-coated films dependent on the PS molecular weight and film thickness are presented. In addition, the solvent content for samples annealed under different conditions is determined. Furthermore, high-resolution NR scans of a distinct sample are compared in terms of long-term behavior. NR curves with an extended $q_{z}$ range are the basis for the analysis with respect to a solvent enrichment in the thin polymer film at the substrate. The corresponding results are exemplarily shown in the last subsection.

3.1. Influence of Polystyrene Molecular Weight. PS films, freshly prepared from protonated toluene and deuterated toluene of a selected molecular weight, are pairwise measured with NR 

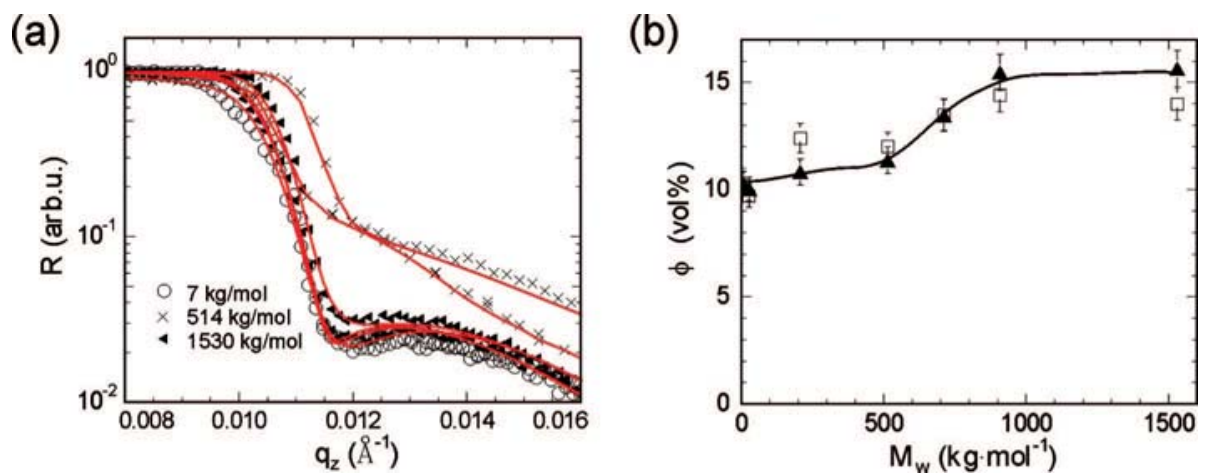

Figure 1. (a) Selection of high-resolution NR scans (symbols) in the region of total external reflection of fresh films of different PS molecular weight $(7,514$, and $1530 \mathrm{~kg} / \mathrm{mol})$ at fixed film thickness. The solid lines are the fits to the measured data for the determination of the total remaining solvent content. (b) Extracted total retained solvent content $\phi(\square)$ shown as a function of the PS molecular weight and the corresponding normalized solvent content $\phi_{\text {norm }}$ of the thin film ( $\left.\mathbf{\Lambda}\right)$. The solid line is a guide to the eye.

for the entire covered range of molecular weights $(7-1530 \mathrm{~kg} /$ mol). This pairwise measurement enables a qualitative comparison during the measurement by comparing the highresolution scans with respect to the expected distinct shift in the critical edge for the deuterated thin film as compared with the protonated thin film. An example of three such pairs is shown in Figure 1a. The use of deuterated toluene significantly shifts the position of the critical edge toward larger $q_{z}$ values as expected, and thus thin films prepared from toluene and toluene- $d_{8}$ can be clearly distinguished. Small differences among the individual curves, for example, a decrease in intensity with increasing $q_{z}$ values, are mainly caused by differences in the film thickness and roughness of the related samples. Therefore, a simple qualitative extraction of the shift in the position of the critical edge is not sufficient, and a quantitative data analysis based on fitting of the NR data is required. This quantitative analysis of the high-resolution NR data is based on a simple single-layer model consisting of the Si substrate and the PS layer on top surrounded by air. Therefore, the components relevant to the analysis are the substrate and the PS layer, which are described by the material-specific SLD, the roughness, and the thickness of the PS layer. In this approach, the SLD of the PS layer, prepared from deuterated toluene, represents the most significant fitting parameter, because it contains the desired information about the solvent content. Therefore, the impact of all other parameters has to be minimized. In this regard, prior to the analysis, the thin films are characterized by XRR to obtain roughnesses and, most importantly, the thickness values, which are not accessible with the NR scans limited to the region of total external reflection. The fits obtained with this model are also shown in Figure 1a (solid lines). Thus, from the fit to the NR data, only the SLD of the PS layer is extracted, from which the (remaining) total solvent content $\phi$ inside the PS films is calculated (by the use of eq 1).

Each NR data point exhibits an error given by the square root of the count rate. These errors result in an error tube around the measured NR curves, which is spanned by the positive and negative deviations (error bars) of each data point. Therefore, each NR curve is virtually shifted toward smaller $q_{z}$, marking the minimum, and toward higher $q_{z}$, marking the maximum, of the position of the critical edge. The respective limits of the position correspond to a minimum and maximum SLD, yielding the error in the extracted SLD. For the presented data, the error in SLD is $\triangle \mathrm{SLD}=0.05 \times 10^{-6} \AA^{-2}$. This error in SLD gives rise to an error in the extracted solvent content, as shown by the error bars in the Figures.

The total solvent content as a function of the PS molecular weight is shown in Figure 1b. Because of the applied layer model, which assumes a layer with thickness, $d$, and an infinitely expanded surface area, $\phi$, is obtained in units of volume percent. To correct for small thickness deviations in the individual samples with respect to the desired mean thickness, we introduce a so-called normalized solvent content referring to one reference thickness

$$
\phi_{\mathrm{norm}}=\phi \frac{d_{\mathrm{m}}}{d_{\mathrm{eff}}}
$$

to decouple the parameters molecular weight and thickness. The mean thickness $d_{\mathrm{m}}=\left\langle d_{\mathrm{eff}}\right\rangle$ is normalized by the individual film thickness $d_{\text {eff }}$, as measured with XRR. Because $d_{\text {eff }}$ is made to be roughly constant for the sample series addressing the molecular-weight dependence, there is no significant change in the observed trend by replacing $\phi$ with $\phi_{\text {norm }}$ (compare open and filled symbols in Figure 1b). However, the behavior of $\phi_{\text {norm }}$ is more continuous, as indicated by the solid line. In general, the normalization will become more significant in the case of larger deviations or intentionally varied values of the effective film thicknesses.

In general, the solvent content increases with increasing molecular weight from 9 to $15 \mathrm{vol} \%$. At molecular weights of $>1000 \mathrm{~kg} / \mathrm{mol}, \phi$ (or $\phi_{\text {norm }}$ ) remains constant within the error. A reason for the molecular-weight-dependent increase in the remaining solvent might be the free volume of the PS films, in which the solvent can be retained. Typically, two different sources contribute to the free volume of a polymer film: (1) an intramolecular contribution, which is the difference between the sum of the atomic volumes and the overall spherical volume spun by the radius of gyration of the individual polymer chain, ${ }^{57}$ and (2) an intermolecular contribution, which is the difference between the overall spherical volume and the volume of the entire thin film. For the reason that the increase in molecular weight is probed at roughly constant film thickness, both contributions increase because of the increased degree of polymerization as well as the radius of gyration. Hence, for thin films with fixed thickness but with increasing molecular weight, the free volume might increase, which acts as free space for the solvent. Of course, the same holds true for the normalized solvent content. With the normalization, the overall area is divided into area occupied by the polymer and free area, which is, by analogy to the free volume, unoccupied area where the solvent can be retained.

3.2. Influence of Film Thickness. For the investigation of the film thickness as the key parameter, the molecular weight was fixed to $M_{\mathrm{w}}(\mathrm{PS})=207 \mathrm{~kg} / \mathrm{mol}$, and the film thickness was varied. For clarity, Figure 2a shows exemplarily PS films prepared from deuterated toluene. For different film thicknesses, a shift in the position of the critical edge in the NR data is 

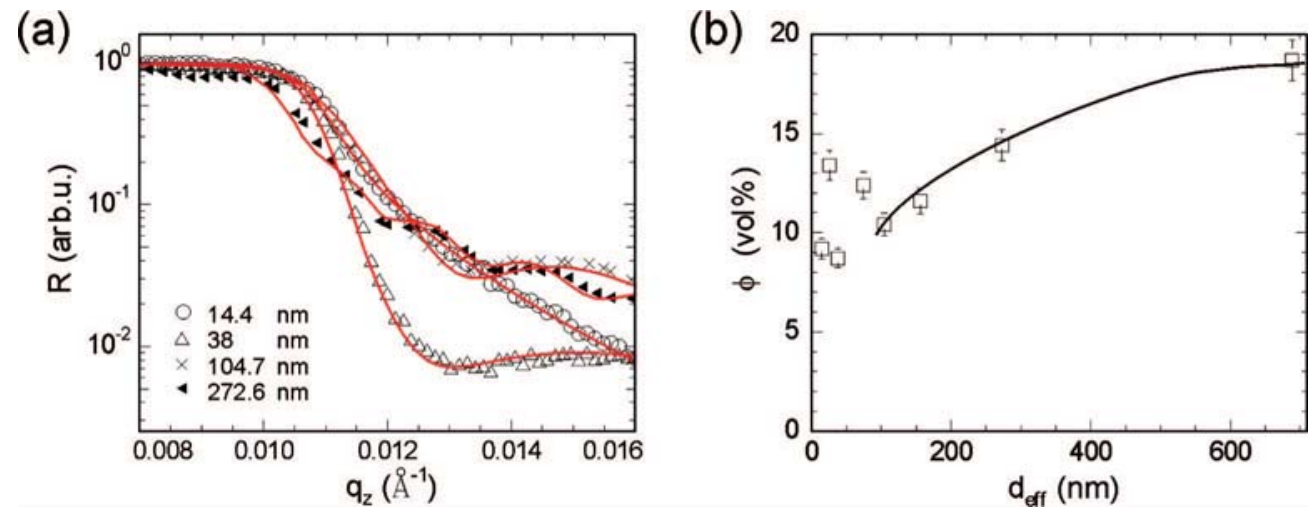

Figure 2. (a) Selection of high-resolution NR scans (symbols) in the region of total external reflection of fresh films of different film thickness at fixed PS molecular weight. The solid lines are the fits to the measured data for the determination of the total remaining solvent content. (b) Extracted

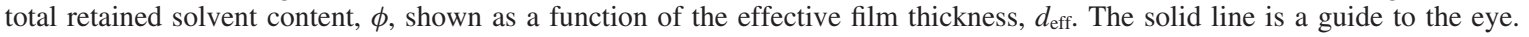

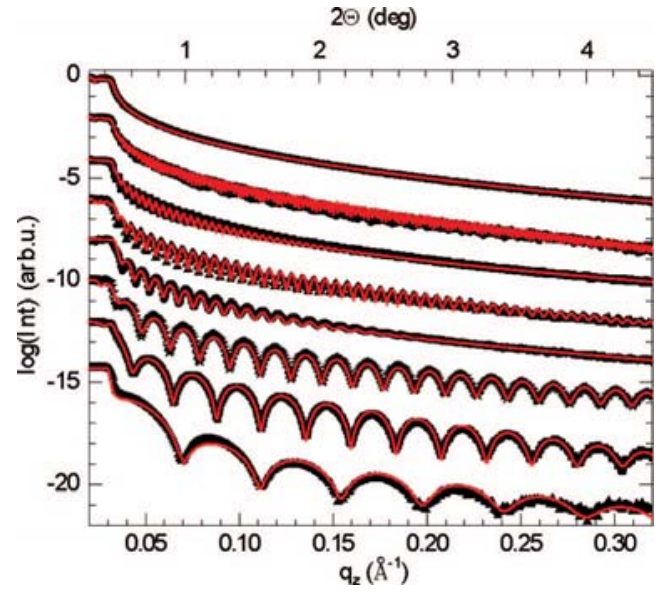

Figure 3. XRR data (symbols) measured for the PS films of different thickness at fixed molecular weight. The solid lines are the fits for determining the thickness, surface, and interface roughness of the film. The intensities are normalized to their maximum intensity. For clarity, the curves are shifted along the intensity axis. From bottom to top, the film thickness increases.

clearly recognizable in Figure 2a. As described already in detail, the data analysis procedure requires the effective film thickness and roughness for the modeling of the NR to extract the SLD. These parameters are again obtained from XRR. The XRR measurements are shown in Figure 3. The extracted effective film thicknesses, $d_{\mathrm{eff}}$, cover a range from $(14.4 \pm 0.2)$ to $(688.1$ $\pm 2.1) \mathrm{nm}$.

With these thicknesses and the obtained roughness values, the single-layer model is again applied to the high-resolution NR scans, and the obtained fits are plotted in Figure 2a. The fits show good agreement with data, and from the respective SLDs, the remaining solvent content is extracted. The solvent content, $\phi$, is plotted as a function of the effective thickness, $d_{\text {eff }}$, in Figure 2b. A calculation of the normalized solvent content, $\phi_{\text {norm }}$, is not meaningful for the control parameter film thickness.

The values of $\phi$ increase with increasing film thickness, although for small film thickness, the determined values of the retained total solvent content, $\phi$, show some scatter all over. With further increasing the film thickness, an approach of a limiting value displaying the PS bulk characteristics is likely.

The all-over increase observed in the retained solvent is the opposite trend as compared with the GC investigation by GarcíaTuriel and Jérome. ${ }^{35}$ However, it might not be a contradiction because García-Turiel and Jérome investigated annealed PS films, whereas our study focuses on untreated films probed directly after spin-coating.
Different film thicknesses are realized by the variation of the polymer concentration in the solution used for spin-coating. Hence, for a thicker film, the amount of solvent per polymer is smaller than that for a thinner film because of the higher PS concentration in the toluene solution. But during preparation (beginning by putting the solution on the substrate), the evaporation at the uppermost part of the film produces a gradient of solvent that is the driving force for the diffusion of solvent molecules toward the polymer/air interface. At the free surface, the solvent evaporates and a more dense and less solventcontaining part is formed on top. Now, for an increased thickness, the diffusion path of solvent molecules from deep inside the film is longer, and less solvent can reach the polymer/ air interface at a given time. Although a smaller amount of solvent is present in the solution that was put on the substrate in the beginning, the increased thickness effect on the evaporation ability prevails, and a higher total solvent content remains for thicker films. In this regard, it has to be emphasized again that the samples are freshly prepared without any further thermal processing. Thus, no annealing is applied to subduct solvent from the spin-coated film. Nevertheless, with this scenario, it is implicitly expected that the silicon/polymer interface will have an influence on the retained solvent as well as result in a gradient of solvent inside the films. From the complex interplay between interaction-driven solvent enrichment at the substrate interface and the possibility of trapping solvent because of limited diffusion, the complex behavior of small film thickness, as seen in Figure 2b, might be explained. The presence of a nonhomogeneous distribution is exemplarily shown in the final section.

3.3. Influence of Annealing and Long-Term Behavior. So far, we report the remaining total solvent content of freshly spincoated films without any postproduction treatment. Thus, nonequilibrium films are probed, and equilibration might be accompanied by a reduced retained solvent content (as suggested by comparison with GC experiments). Moreover, polymer films are typically cured at selected temperatures to reduce the solvent content. For many applications, long-term behavior comprising the aging caused by a change in the remaining solvent is important. Therefore, typically, one wants to remove the remaining solvent, regardless of the present amount, and hence enable the relaxation of the polymer chains to equilibrium. ${ }^{58-61}$ In literature, innumerable publications describe the application of thermal annealing (lower or higher than $T_{\mathrm{g}}$ of polymer) on polymer thin films as a part of the sample preparation. ${ }^{58-61}$

To address these points, a series of PS films of fixed film thickness and molecular weight $\left(M_{\mathrm{w}}(\mathrm{PS})=207 \mathrm{~kg} / \mathrm{mol}\right)$ was investigated. The spin-coating of the films was followed by a postproduction treatment. One reference sample was always left untreated to allow for direct comparison. As postproduction 

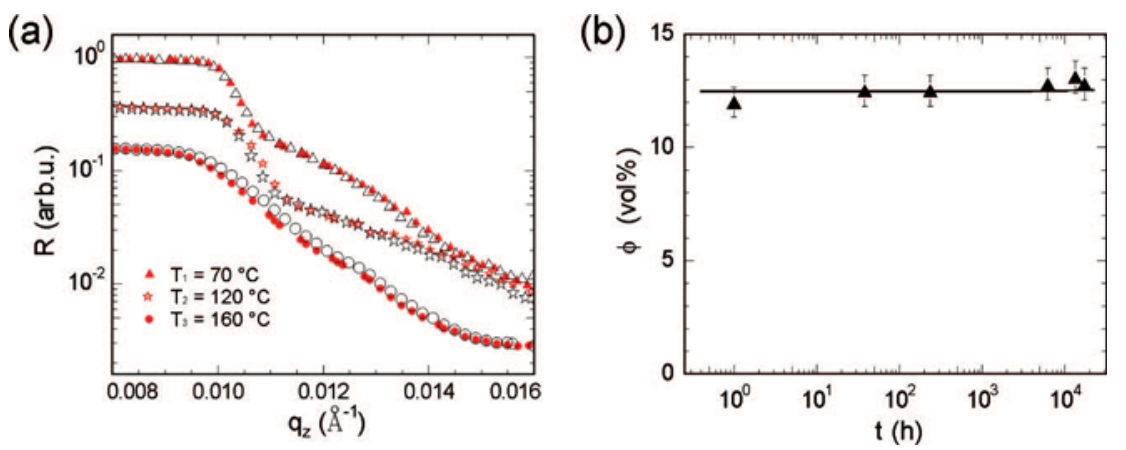

Figure 4. (a) High-resolution NR data in the region of total external reflection of thin films as prepared (open symbols) and after annealing (closed symbols) for $8 \mathrm{~h}$ at $70(\triangle, \mathbf{\Delta}), 120\left({ }^{\star}, \star\right)$, and $160^{\circ} \mathrm{C}(\mathrm{O}, \mathbf{O})$. For clarity, the curves are shifted along the reflectivity axis. (b) Long-term behavior of the total retained solvent content $\phi$ as a function of the time, $t$, since preparation. The solid line is a guide to the eye.

treatment, three different annealing procedures were chosen: The PS films were annealed for $8 \mathrm{~h}$ in vacuum $\left(p \approx 5 \times 10^{-2} \mathrm{mbar}\right)$ at different temperatures, $T_{1}=70^{\circ} \mathrm{C}\left(T_{1}<T_{\mathrm{g}}(\mathrm{PS})\right), T_{2}=120$ ${ }^{\circ} \mathrm{C}\left(T_{2}>T_{\mathrm{g}}(\mathrm{PS})\right)$, and $T_{3}=160{ }^{\circ} \mathrm{C}\left(T_{3} \gg T_{\mathrm{g}}(\mathrm{PS})\right)$.

After annealing, the thin films were examined with optical microscopy to rule out any destruction, for example, due to dewetting of the PS film from the silicon surface. The asprepared samples were immediately measured with NR, followed by the annealed samples. The respective high-resolution scans of the as-prepared and annealed samples are plotted in Figure 4a. Annealing at temperature $T_{1}$ leads to no change at all, and thus no effect of the annealing is observable, and the solvent content remains constant at $\sim 13$ vol $\%$. This is caused by the fact that $T_{1}$ is well below the glass-transition temperature of PS $\left(T_{\mathrm{g}}(\mathrm{PS}) \approx 100{ }^{\circ} \mathrm{C}\right)$. For the annealing temperature $T_{2}$, only a minor change is observed, resulting in a decrease in the solvent content by only 1.0 vol \% because the selected temperature is only slightly above $T_{\mathrm{g}}(\mathrm{PS})$. For both, the XRR measurement indicates that the annealed film is $0.3 \mathrm{~nm}$ thinner than the as-prepared film, whereas this deviation lies within the error of the effective thickness. However, because the duration of annealing was limited to $8 \mathrm{~h}$, a much longer annealing at $T_{2}$ might result in a sufficient reduction in the solvent content. As an alternative to an increased annealing time at temperature $T_{2}$, we increase the annealing temperature. For this even higher annealing temperature $T_{3}$, the NR measurement of the annealed film clearly differs from the as-prepared film. The annealing results in a decrease in the amount of solvent by $3.2 \mathrm{vol} \%$. An indication of the removal of solvent is also found with the XRR measurement because the thickness obtained changes by 0.7 $\mathrm{nm}$, which is outside the error for $d_{\mathrm{eff}}$ and thus can be attributed to the annealing effect. Nevertheless, obviously, the selected conditions are not suitable for the complete removal of the solvent. Thus, typically, considerably longer annealing times might be required for decreasing the total remaining solvent content more strongly. Therefore, compared with the annealing condition chosen by García-Turiel and Jérome $\left(115^{\circ} \mathrm{C} \text { for } 6 \mathrm{~h}\right)^{35}$ it appears to be unlikely that their observed decrease in solvent content with film thickness can be easily translated to our observed increase in film thickness by taking annealing into account. It might be more likely that the difference is caused by the applied experimental method (GC versus NR).

In addition to annealing conditions, the long-term behavior has been addressed over 2 years. During several beamtimes within the past 2 years, for one selected sample $\left(M_{\mathrm{w}}(\mathrm{PS})=\right.$ $\left.207 \mathrm{~kg} / \mathrm{mol}, d_{\text {eff }}=(63.1 \pm 0.4) \mathrm{nm}\right)$, high-resolution NR has been measured. This long-term investigation covers a period of $\sim 17280 \mathrm{~h}$ while including six measurements, starting with the freshly prepared thin film with an age of $\sim 1 \mathrm{~h}$. In between measurements, the sample was stored in a box (to protect against dust) in a dark and dry place at room temperature. Annealing

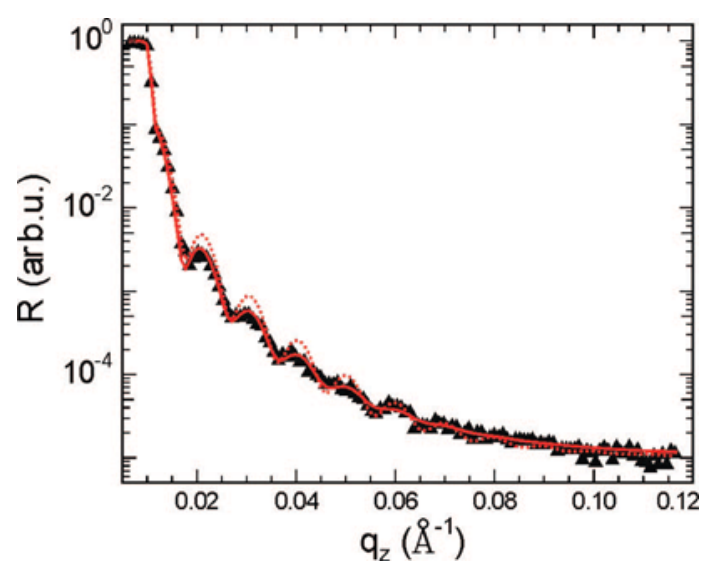

Figure 5. Example of NR data (symbols) of a freshly prepared PS film (from toluene- $d_{8}, M_{w}(\mathrm{PS})=207 \mathrm{~kg} / \mathrm{mol}$, and $d_{\mathrm{eff}}=63.1 \mathrm{~nm}$ ). The lines show fits to the measured data on the basis of the singlelayer model $(\cdots)$ and an advanced double-layer model (-), assuming a thin enrichment layer at the polymer/substrate interface.

of a PS film over such a long period turned out to be impossible because of degradation of the film, for example, by dewetting. The total solvent content was extracted following the procedure explained above and plotted as a function of the storage time since preparation $t$ in Figure $4 \mathrm{~b}$. No changes in the effective film thickness were detected, and thus the normalized solvent content shows the identical behavior. In the full period of 2 years, the solvent content in the PS film remains constant at $\sim 12.6 \mathrm{vol} \%$. Therefore, no aging in terms of changing the retained solvent is verifiable, and no mechanical alteration of the thin PS film is expected, which is supported by the optical investigation that also show no changes.

3.4. Solvent Enrichment at Polymer/Substrate Interface. So far, the analysis focused on the remaining total solvent content, assuming a homogeneous layer consisting of PS and deuterated or protonated solvent, respectively. As mentioned in the introductory part, there is the reasonable characteristic of a solvent enrichment at the polymer/substrate interface induced by the evaporation, which takes place at the polymer/ vapor interface. Therefore, depletion of the solvent in regions near the surface occurs. ${ }^{36-38}$ In particular, one would expect a nonhomogenous solvent distribution inside the film, that is, increasing solvent concentration with depth. Figure 5 shows an example of an extended NR measurement of a thin PS film prepared from toluene- $d_{8}$ with $M_{\mathrm{w}}(\mathrm{PS})=207 \mathrm{~kg} / \mathrm{mol}$ and $d_{\text {eff }}$ $=(63.1 \pm 0.7) \mathrm{nm}$.

Simply applying the single-layer model with thickness and interface roughness as obtained from the XRR measurements yields a good fit for only the $q_{z}$ region around the critical edge, but a deviation from the measured data at the large $q_{z}$ values is 
observed. Herewith, a remaining total solvent content of $13 \mathrm{vol}$ $\%$ was extracted for the thin film. However, the simple singlelayer model is not sufficient for describing the full NR curve. In an alternative approach to fit the NR data, the roughness of the Si substrate was included in the free fitting parameters, which resulted in a roughness increase by a factor of 10 for the improved fit. However, because the roughness of $\mathrm{Si}$ wafer substrates is known to be on the order of a few angstroms and significantly well characterized with XRR, the resulting bestfit model parameters are basically of no physical meaning. Nevertheless, because an increased amount of solvent (as one would find in an enrichment layer) yields an increase in SLD, therewith approaching the SLD of the Si substrate, the result can be interpreted as a kind of gradient from high SLD (substrate) toward lower SLD of the polymer film. Consequently, from these results it can be concluded, that the introduction of an additional layer to the polymer/substrate interface transforms our findings into a reasonable physical model.

The introduction of the double-layer model includes the concept of a solvent enrichment at the substrate/polymer interface. By fitting this model to the measured data, a considerable improvement is observed, as can be seen in Figure 5. From the obtained SLDs and thicknesses by the best fit, we extracted a solvent content of 25.9 vol \% for the enrichment layer with a thickness of $\sim 4 \pm 0.5 \mathrm{~nm}$ near the Si substrate. The remaining part of the PS film had a thickness of $60 \pm 0.7$ $\mathrm{nm}$ and a solvent content of only $3.2 \mathrm{vol} \%$. This clearly verifies a solvent enrichment at the substrate/polymer interface and is in good agreement with conclusions of other groups that the solvent is retained at the polymer/substrate interface. ${ }^{29-35}$ Moreover, a density decrease in PS films was detected, for example, by Jonar and coworkers, ${ }^{38}$ extending over a region of two to three statistical segment lengths at the solid interface, which also agrees well with the idea that in this region the polymer is depleted and the solvent enriched.

However, for many experiments, the exact shape of the solvent profile along the surface normal inside the polymer film will be not of utmost importance rather than the knowledge about the total solvent content. The method used in this work, combining selective deuteration with high-resolution NR is, of course, not restricted to the examined model system PS/toluene but can be expanded to other technological relevant systems.

The amount of remaining solvent detected as well as its detailed distribution inside the polymer film (layering) has an important interplay with microscopic and macroscopic properties of thin polymer films. For example, recent experiments focusing on molecular dynamics in thin polymer layers with a free upper interface revealed no shifts in the average relaxation time and no broadening of the dynamic glass-transition for PS down to film thicknesses of $\sim 10 \mathrm{~nm} .{ }^{62}$ Therefore, the decoupling of confinement effects ${ }^{63}$ from the change in the solvent content per the area of the polymer film might be necessary. For applications, it is comforting that the total remaining solvent in the polymers remains unchanged over long amounts of time (up to years) if no postproduction treatment is applied. If the polymer films are thermally processed, then application can benefit from the knowledge that the total solvent content is not altered as long as temperatures stay below the glass-transition temperature of the polymer.

\section{Conclusions}

The detection of remaining solvent in thin polymer films is of importance because of its effect on chain mobility and film homogeneity. Moreover, it is crucial information for all swelling experiments to define the initial dry state of the polymer film.
Using NR, we probed the remaining solvent content in thin, freshly spin-coated polymer films. We focused on the wellestablished model system of PS spin-coated from toluene onto $\mathrm{Si}$ substrates. The investigation addresses key parameters such as the molecular weight of the polymer, the film thickness of the polymer films, different annealing conditions, and long-term aging. It was observed that a significant and not negligible amount of solvent remains in the freshly prepared films, for example, depending on the molecular weight 9 to $15 \mathrm{vol} \%$ for a PS film of $50 \mathrm{~nm}$ thickness. This total remaining solvent content increases with increasing PS molecular weight as well as with increasing PS film thickness. The observed molecular weight dependence might be explained by the increase in free volume with increasing molecular weight. The observed film thickness dependence contradicts observations based on GC measurements, which, only to some extent, might be due to the different applied experimental protocols. Moreover, the storage capacity of the PS films is strongly influenced by the presence of the Si substrate. Closer to the Si/polymer interface, a larger volume fraction of solvent can remain inside the film, whereas with increasing distance to the substrate, this value approaches a bulk value. This behavior is supported by the detailed analysis of a solvent profile in the PS films. In a thin part of the PS film close to the substrate, solvent is significantly enriched, whereas in the upper rest of the polymer films, a value close to the bulk value is reached.

The additional investigation of the effect of annealing on the solvent content reveals that temperatures higher than $T_{\mathrm{g}}$ and the boiling temperature of the solvent are most suitable for the efficient removal of the solvent. As presented, the selected conditions were not suitable for removing all remaining solvent. Only a reduction in solvent in the film can be achieved. It is expected that an increase in annealing time would yield a further reduction. However, we have shown that a dry film is actually not a dry film at all, as assumed in many investigations for asprepared as well as annealed films.

We must point out that the observed behavior for different molecular weight and film thickness as well as the effect of annealing and the long-term characteristics might be different for more complex model systems, for example, block copolymer, polymer nanocomposite, or polymer blend thin films. In terms of NR, such systems require a more advanced layer model for analysis and thus detailed complementary characterization beforehand. In addition to the solvent-retaining mechanisms discussed in this investigation, further mechanisms might be responsible for the residual solvent inside the thin spin-coated films; therefore, NR will show a nontrivial behavior.

Acknowledgment. We thank B. Russ and P. Böni for their assistance and support in the X-ray reflectivity experiments. The financial support by DFG (grant MU1487/4-2) is gratefully acknowledged.

\section{References and Notes}

(1) Yada, T. Jpn. J. Appl. Phys 1998, 37, 2752.

(2) Cecchi, M.; Smith, H.; Braun, D. Synth. Met. 2001, 121, 1715.

(3) Jeong, S.; Jang, W.-H.; Moon, J. Thin Solid Films 2004, 466, 204.

(4) Müller-Buschbaum, P.; Gebhardt, R.; Maurer, E.; Bauer, E.; Gehrke, R.; Doster, W. Biomacromolecules 2006, 7, 1773.

(5) Hall, D. H.; Underhill, P.; Torkelson, J. M. Polym. Eng. Sci. 1988, 38, 2039.

(6) Bornside, D. E.; Macosko, C. W.; Scriven, L. E. J. Imaging Sci. Technol. 1987, 13, 122.

(7) Bornside, D. E.; Macosko, C. W.; Scriven, L. E. J. Appl. Phys. 1989, $66,5185$.

(8) Dusková-Smrcková, M.; Dusek, K. J. Mater. Sci. 2002, 37, 4733.

(9) Norrman, K.; Ghanbari-Siahkali, A.; Larsen, N. B. Annu. Rep. Prog. Chem., Sect. C 2005, 101, 174.

(10) Croll, S. G. J. Appl. Polym. Sci. 1979, 23, 847. 
(11) Brulet, A.; Boué, F.; Menelle, A.; Cotton, J. P. Macromolecules 2000, 33, 997.

(12) Mundra, M. K.; Ellison, C. J.; Behling, R. E.; Torkelson, J. M. Polymer 2006, 47, 7747 .

(13) Cohen, M. H.; Turnbull, D. J. Chem. Phys. 1959, 31, 1164.

(14) Lee, S.-H.; Kang, H.; Kim, Y. S.; Char, K. Macromolecules 2003, $36,4907$.

(15) Graves-Abe, T.; Pschenitzka, F.; Jin, H. Z.; Bollman, B.; Sturm, J. C.; Register, R. A. J. Appl. Phys. 2004, 96, 7154.

(16) Thompson, R. L.; McDonald, M. T.; Lenthall, J. T.; Hutchings, L. R. Macromolecules 2005, 38, 4339.

(17) Tsige, M.; Grest, G. S. J. Phys.: Condens. Matter 2005, 17, S4119.

(18) Extrand, C. W. Langmuir 1993, 9, 475.

(19) Strawhecker, K. E.; Kumar, S. K.; Douglas, J. F.; Karim, A. Macromolecules 2001, 34, 4669.

(20) To, T.; Wang, H.; Djurisic, A. B.; Xie, M. H.; Chan, W. K.; Xie, Z.; Wu, C.; Tong, S. Y. Thin Solid Films 2004, 467, 59.

(21) Huang, Y.; Paul, D. R. Macromolecules 2005, 38, 10148.

(22) DeMaggio, G. B.; Frieze, W. E.; Gidley, D. W.; Zhu, M.; Hristov, H. A.; Yee, A. F. Phys. Rev. Lett. 1997, 78, 1524.

(23) Prucker, O.; Christian, S.; Bock, H.; Rühe, J.; Frank, C. W.; Knoll, W. Macromol. Chem. Phys. 1998, 199, 1435.

(24) Forrest, J. A.; Dalnoki-Veress, K. Adv. Colloid Interface Sci. 2001, 94, 167.

(25) Grohens, Y.; Hamon, L.; Reiter, G.; Soldera, A.; Holl, Y. Eur. Phys. J. E 2002, 8, 217.

(26) Richardson, H.; Sferrazza, M.; Keddie, J. L. Eur. Phys. J. E 2003, $12, \mathrm{~S} 87$.

(27) Zhao, W.; White, J. M. Appl. Phys. Lett. 2007, 90, 181906.

(28) Grohens, Y.; Hamon, L.; Spevacek, J.; Holl, Y. Macromol. Symp. 2003, 203, 155.

(29) Arney, J. S.; Pollack, L. B. J. Am. Inst. Conserv. 1980, 19, 69.

(30) Kumar, N.; Gow, J. G. J. Chromatogr., A 1994, 667, 235.

(31) Incavo, J. A.; Jain, V.; Qi, J. S.; Krishnan, C. Chromatographia 1996, $43,31$.

(32) Bistac, S.; Schultz, J. Prog. Org. Coat. 1997, 31, 347.

(33) Kelly, D. M.; Vos, J. G.; Hillmann, A. R. J. Mater. Chem. 1997, 7, 913.

(34) Pan, L.; Zhang, M.; Nakayama, Y. J. Chem. Phys. 1999, 110, 10509.

(35) García-Turiel, J.; Jérome, B. Colloid Polym. Sci. 2007, 285, 1617.

(36) Lee, L. T.; Guiselin, O.; Lapp, A.; Farnoux, B. Phys. Rev. Lett. 1991, 67, 2838
(37) Vrentas, J. S.; Vrentas, C. M. J. Appl. Polym. Sci. 1996, 60, 1049.

(38) Bollinne, C.; Stone, V. W.; Carlier, V.; Jonas, A. M. Macromolecules 1999, 32, 4719.

(39) Penfold, J.; Thomas, R. K. J. Phys.: Condens. Matter 1990, 2, 1369.

(40) Zabel, H. Appl. Phys. A: Mater. Sci. Process. 1994, 58, 159.

(41) Geoghegan, M.; Boué, F.; Bacri, G.; Menelle, A.; Bucknall, D. G. Eur. Phys. J. B 1998, 3, 83.

(42) Penfold, J. Curr. Opin. Colloid Interface Sci. 2002, 7, 139.

(43) Müller-Buschbaum, P.; Bauer, E.; Maurer, E.; Cubitt, R. Physica B 2006, 385-386, 703 .

(44) Müller-Buschbaum, P.; Bauer, E.; Maurer, E.; Nelson, A.; Cubitt, R. Phys. Status Solidi RRL 2007, 1, R68.

(45) Kanaya, T.; Miyazaki, T.; Watanabe, H.; Nishida, K.; Yamano, H.; Tasaki, S.; Bucknall, D. B. Polymer 2003, 44, 3769.

(46) Spangler, L. L.; Torkelson, M.; Royal, J. S. Polym. Eng. Sci. 1990, 30,644 .

(47) Paniez, P.; Pons, M.; Joubert, O. Microelectron. Eng. 1990, 11, 469.

(48) Liu, J.; Deng, Q.; Jean, Y. C. Macromolecules 1993, 26, 7149.

(49) Yu, Z.; Yashi, U.; McGervey, J. D.; Jamieson, A. M.; Simha, R. J. Polym. Sci., Part B: Polym. Phys. 1994, 32, 2637.

(50) Hong, S.-U. Ind. Eng. Chem. Res. 1995, 34, 2536.

(51) Algers, J.; Suzuki, R.; Ohdaira, T.; Maurer, F. H. J. Polymer 2004, $45,4533$.

(52) Müller-Buschbaum, P. Euro. Phys. J. E 2003, 12, 443.

(53) Schubert, D. W. Polym. Bull. 1997, 38, 177.

(54) Georgii, R.; Böni, P.; Janoschek, M.; Schanzer, C.; Valloppilly, S. Physica B 2007, 397, 150.

(55) Parrat, L. G. Phys. Rev. 1954, 95, 359.

(56) Nelson, A. J. Appl. Crystallogr. 2006, 39, 273.

(57) Zhou, Z.; Yan, D. Macromol. Theory Simul. 1997, 6, 597.

(58) Guerrier, B.; Bouchard, C.; Allain, C.; Bénard, C. AIChE J. 1998, 44, 791.

(59) Romdhane, I. H.; Price, P. E.; Miller, C. A.; Benson, P. T.; Wang, S. Ind. Eng. Chem. Res. 2001, 40, 3065.

(60) Yamamura, M.; Horiuchi, K.; Kajiwara, T.; Adachi, K. AIChE J. 2002, $48,2711$.

(61) de Gennes, P. G. Eur. Phys. J. E 2002, 7, 31.

(62) Serghei, A.; Huth, H.; Schick, C.; Kremer, F. Macromolecules 2008, $41,3636$.

(63) Serghei, A.; Tress, M.; Kremer, F. Macromolecules 2006, 39, 9358. 